\title{
球形移动机器人的研究进展与发展趋势*
}

\author{
战 强 1 李 伟 1,2 \\ (1. 北京航空航天大学机器人研究所 北京 100191; \\ 2. 北京航空航天大学中法工程师学院 北京 100191)
}

\begin{abstract}
摘要: 球形移动机器人(简称球形机器人)是一种拥有球形外壳的全封闭机器人, 它通过质心偏移或者动量守恒等原理来实现 运动。该类机器人具有良好的密封性, 平衡性强且运动灵活性高, 不存在侧翻问题, 因此球形机器人在星球探索、危险环境 探测等领域具有较大优势和广泛应用前景。然而球形机器人具有非完整约束、欠驱动、非链式等特点, 导致其运动控制问题 非常复杂且常规控制方法无法应用到它的运动控制上, 阻碍了球形机器人的应用和发展。对国内外在球形机器人的机构设计、 运动学和动力学建模方法以及控制方法等方面的研究进展进行回顾、综述与分析, 其中也包括作者所在实验室对 BHQ 系列 球形机器人研究工作的全面回顾; 按照驱动方式对球形机器人进行归纳分类, 并从运动学建模、动力学建模、开环控制和闭 环控制四个方面介绍球形机器人研究的关键技术和发展趋势; 最后针对球形机器人的研究进展和发展趋势给出五点结论。 关键词: 球形机器人；机构设计；运动学模型；动力学模型；控制方法
\end{abstract} 中图分类号: TP242

\section{Research Progress and Development Trend of Spherical Mobile Robots}

\author{
ZHAN Qiang ${ }^{1} \quad$ LI Wei $^{1,2}$
}

(1. Robotics Institute, Beihang University, Beijing 100191;

2. Ecole Centrale de Pékin, Beihang University, Beijing 100191)

\begin{abstract}
Spherical mobile robot (spherical robot in short) is a fully enclosed robot with a ball-shaped outer shell, which can move by shifting its gravity center or through the principle of momentum conservation. Spherical robot has the characteristics of good sealability, high moving flexibility and strong stability as a roly-poly. Thus spherical robot has big advantages and wide future applications in the exploration of outer planets and the detection of dangerous environments. However, spherical robot is a special type of nonholonomic, under-actuated and unchained system, which makes its motion equations very complicated and the conventional methods cannot be applied to control it. This hinders the development and application of spherical robots. Review, summary and analysis of spherical robots' research progress on mechanism design, kinematics modeling, dynamics modeling, control methods and so on are given, including the comprehensive review of the BHQ series spherical robots developed by the authors' laboratory. The spherical robots are classified according to the driving modes. The key technologies and development trends of the research on spherical robots are introduced from four aspects: kinematics modeling, dynamic modeling, open-loop control and closed-loop control. At last, five conclusions about the research progress and development trend are given.
\end{abstract}

Key words: spherical robot; mechanism design; kinematic model; dynamic model; control method

\section{0 前言}

随着科学技术的发展, 机器人在人类的生活和 工作中扮演着越来越重要的角色, 其中, 移动机器 人是机器人领域的一个重要分支。传统的轮式、履 带式移动机器人发展较早, 目前技术较为成熟并且 应用广泛。随着机器人应用领域的不断扩大, 新型

* 国家自然科学基金资助项目(50705003)。20180306 收到初稿, 20181029 收到修改稿
的移动机器人也在逐步出现, 成为传统移动机器人 的有益补充。

球形机器人是近二十年前才出现的一种新型的 移动机器人。球形机器人一般拥有全封闭球形外壳, 球壳内部包含控制系统、动力系统、运动执行装置、 传感器等, 它通过质心偏移、动量守恒等原理实现 运动。与传统移动机器人相比, 球形机器人具有良 好的密封性, 平衡性强且运动灵活, 而且可以像倒 立摆一样不存在侧翻问题。另外, 球形机器人可以 利用球形外壳通过滚动对冲击力进行有效缓冲, 从 
而使机器人的内部结构得到保护。因此, 球形机器 人在星球探索、危险环境探测、管道内部探测等领 域具有显著优势和广泛应用前景。

球形机器人在进行环境探测时, 需要保证机器 人内部的摄像机等机载设备的稳定性, 并且可以按 照遥控指令或沿着规划的路径运动, 这就要求球形 机器人具有良好的运动可控性、稳定性、实时性以 及较高的运动精度。然而, 球形机器人具有非完整 约束、欠驱动、非链式、强耦合等特点, 并且具有 震荡的倾向性, 导致其理论模型非常复杂, 球形机 器人的运动控制一直是不易解决的难题。另外, 传 统的非完整约束控制理论大多是针对链式系统研究 的, 但是球形机器人模型无法转化为链式系统, 使 得相关理论难以应用于球形机器人的运动控制, 这 更加阻碍了球形机器人的应用和发展。

因此, 设计出拥有良好结构特性的球形机器人 并且对其实现精确的运动控制具有重要的理论研究 意义和工程应用价值。球形机器人的设计和控制问 题一直吸引着国内外各实验室的众多研究人员, 是 当今机器人领域的一个研究热点。

本文对自 20 世纪 90 年代球形机器人出现以来 国内外的研究进展进行了总结。由于国外的研究开 始略早且球形机器人种类更为全面, 因此首先基于 各种类型球形机器人出现时间的先后顺序分类介绍 了国外的研究进展, 然后对作者所在实验室对球形 机器人近二十年的研究成果进行了介绍, 随后对国 内其他研究工作进行了介绍。在对国内外研究现状 进行全面回顾之后, 本文按照驱动方式对球形机器 人进行归纳分类, 并从运动学建模、动力学建模、 开环控制和闭环控制四个方面介绍了球形机器人研 究的关键技术和发展趋势, 分析了其在应用上的难 点, 并结合机器人新技术的发展趋势对球形机器人 进行了展望。最后, 针对球形机器人的研究进展和 发展趋势给出五点结论。

\section{1 国外研究进展}

1996 年, 芬兰赫尔辛基科技大学 (Helsinki University of Technology)的 HALME 等 ${ }^{[1]}$ 研制了一 款小车驱动的球形机器人 Rollo, 其模型如图 1 所 示。该机器人靠一个在球壳内部滚动的独轮小车驱 动, 主动轮通过转动沿球壳内壁攀爬, 从而改变机 器人质心, 驱动球壳向前滚动。HALME 等主要考 虑了机器人的直线运动, 仅对球形机器人的前向滚 动建立了动力学模型。独轮车驱动结构不稳定, 因 此很难实现对该机器人位置的精确控制, 但是以一
定的平均速度按照预期轨迹运动是可行的。

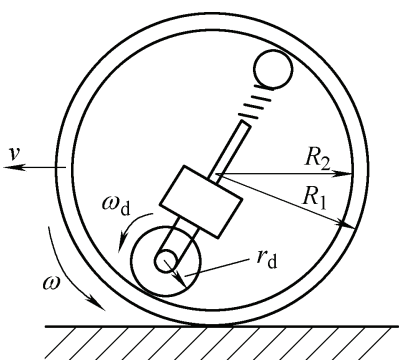

图 1 HAMEL 等研制球形机器人的模型 ${ }^{[1]}$

同样利用小车驱动, 1997 年, 意大利比萨大 学(Università di Pisa)的 BICCHI 等 ${ }^{[2]}$ 设计了一款球 形机器人 Sphericle(图 2)。该机器人球壳内部放有 一辆四轮小车, 小车运动带动整个球形机器人运 动。由于小车与球壳之间也存在非完整约束, 这 使系统的动力学模型更加复杂, 加大了对机器人 进行控制的难度。2010 年, 美国的 BERNSTEIN 等创建了球形玩具机器人公司 Sphero ${ }^{[3]}$, 研发了一 款可遥控球形玩具机器人 $S P K R+($ 图 3), 是靠内部 二轮小车驱动。

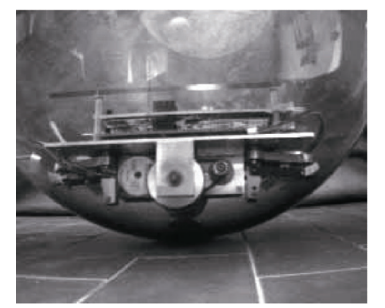

图 2 Sphericle 球形机器人样机 ${ }^{[2]}$

同样利用小车驱动, 2015 年，俄罗斯 Izhevsk State Technical University 的KARAVAEV 等 ${ }^{[4]}$ 研制了 一款球形机器人(图 4), 其内部小车由三个麦克纳姆 轮支撑。他们假设内部小车与球之间以及球壳与地 面之间均没有滑动摩擦, 建立了机器人的运动学模 型, 对机器人进行运动控制。但是随着机器人速度 的增加, 偏移量也会增加, 这限制了其应用, 因此 他们建立了该机器人的拉格朗日动力学模型, 并提 出了基于动力学的轨迹跟踪控制算法, 在理论上对 其直线运动时的稳定性进行了证明。

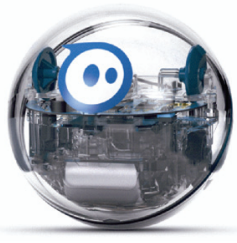

图 $3 \mathrm{SPRK}+{ }^{[3]}$

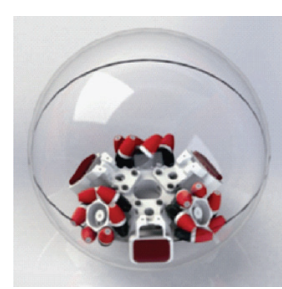

图 4 KARAVAEV 的机器人样机 ${ }^{[4]}$
1999 年, 美国卡耐基梅隆大学(Carnegie Mellon University)的 CHEMEL 等 ${ }^{[5]}$ 研制了一种球形移动机 
器人, 通过改变机器人球壳球心下方质量块的重力 势能来驱动机器人滚动, 同时可以基于角动量守恒 原理实现原地转向。

1999 年, 美国密执安州立大学(Michigan State University)的 MUKHERJEE ${ }^{[6]}$ 等提出了一种球形机 器人的驱动方案, 如图 5 所示, 机器人内部含有四 根螺杆, 其中每根螺杆上装有一个质量块并配有独 立的驱动系统, 机器人通过改变四个质量块的位置 来改变机器人整体的质心位置, 从而产生偏心力矩 来实现机器人的全方位运动。2012 年, MUKHERJEE 等 ${ }^{[7]}$ 优化了该设计并制作了一款结构 类似的球形机器人, 通过三个电动机分别控制三个 质量块的位置来驱动机器人运动。基于同样的驱动 原理, 伊朗 Azad University of Qazvin 的 JAVADI 等 ${ }^{[8]}$ 研发了 August 球形机器人。

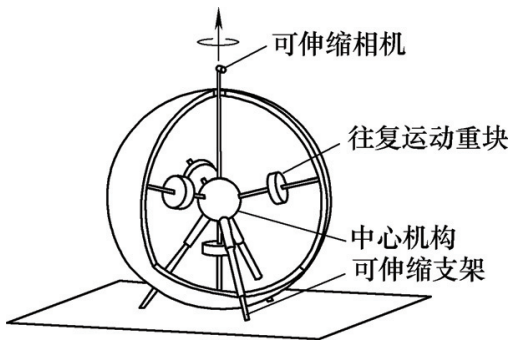

图 5 MUKHERJEE 等设计的球形机器人结构图 ${ }^{[6]}$

2000 年, 美国特拉华大学 (University of Delaware)的 BHATTACHARYA 等 ${ }^{[9]}$ 利用角动量守 恒原理设计了一种球形机器人的驱动方案。球体内 部装有两个相互垂直的转盘, 分别用两个电动机控 制。他们建立了机器人的动力学方程。机器人在运 动的过程中会一直保持重心位于球体形心与地面 接触点之间, 因此机器人没有受到重力矩; 另外地 面对机器人的反作用力都作用于接触点上, 因此机 器人在地面接触点处受到的外部力矩为零, 这样得 到关于地面接触点的三个动量矩守恒方程。另外在 球壳与地面之间存在两个非完整约束方程, 因此总 共有五个约束方程。机器人状态可以由五个独立自 由度来表示, 其中两个表示位置, 三个表示姿态。 五个约束方程对应五个自由度, 因此该系统描述方 程组存在唯一解析解。利用该方法建立起的动力学 方程较为复杂, BHATTACHARYA 等只分析了系统 的开环响应, 并且通过试验验证了模型的准确性。 另外, BHATTACHARYA 等提出了基于最低能耗和 基于最短时间的两种非完整约束系统的轨迹规划 方法。2010 年, 印度理工学院孟买校区(Indian Institute of Technology Bombay)的 JOSHI 等 ${ }^{[10-11]}$ 同 样利用角动量守恒原理制作了一款类似的球形机 器人(图 6)。

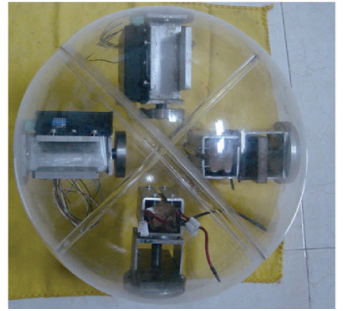

图 6 JOSHI 等研制的球形机器人样机 ${ }^{[11]}$

同样利用角动量守恒原理, 2015 年, 印度理工 学院马德拉斯校区(Indian Institute of Technology Madras)的 Vijay MURALIDHARAN 等 ${ }^{[12]}$ 对一个由 三个独立电动机驱动的球形机器人(图 7)进行了研 究。他们建立了机器人的运动学模型, 并利用 Euler-Poincaré 方程建立了机器人姿态的动力学模 型, 在此基础之上构建了新的控制系统, 并利用李 代数秩条件证明了控制系统的强可达性和小范围局 部稳定性, 利用李雅普诺夫稳定性原理证明了其在 近乎全局上的渐进稳定性。最终, 他们提出了对机 器人位姿的稳定控制方法和对于机器人位置的轨迹 跟踪控制方法。

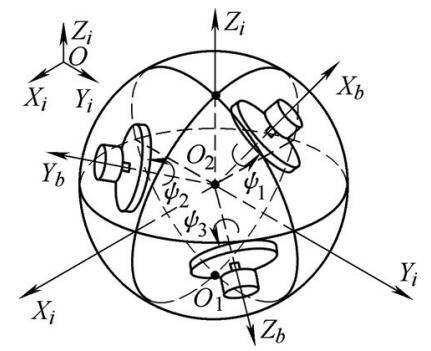

图 7 MURALIDHARAN 等的球形机器人模型 ${ }^{[12]}$

2005 年, 日本立命馆大学(Ritsumeikan University) 的 SUGIYAMA 等 ${ }^{[13]}$ 研制了一款通过球体外壳形变 来运动的球形机器人(图 8)。机器人内部由多根记忆 合金支撑，并且每根记忆合金都由单独的电压信号 控制, 通过改变电压信号的大小来使各根合金产生 相应的形变, 机器人外壳产生规律变化从而来实现 滚动。另外, 当合金形变量很大时, 球体可以通过 形变的瞬间恢复来完成跳跃。同样利用形变原理, 2010 年, 美国范德堡大学(Vanderbilt University)的 WAIT 等 ${ }^{[14]}$ 研发了一款类似于足球的球形机器人。机 器人外部由许多气囊组成, 通过对各个气囊的充放气 来完成球体外壳的形变从而推动机器人前行(图 9)。

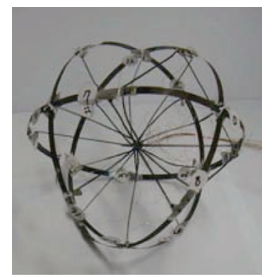

图 8

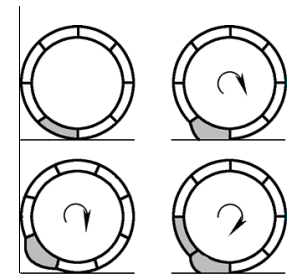

图 9 足球机器人的运动 ${ }^{[14]}$ 
2006 年, 日本神户大学(Kobe University)的 OTANI 等 ${ }^{[15]}$ 研制了一款具有陀螺运动特性的球形 机器人(图 10)。该机器人由三个电动机驱动, 其中 一个电动机控制陀螺的高速转动, 另外两个电动机 位于陀螺仪机箱(Gyro Case)内的两个转轴处, 它们 提供转矩使陀螺仪机箱绕与陀螺转轴垂直的轴转 动。他们建立了该机器人的运动学模型和动力学模 型, 并利用李括号证明了机器人基于运动学模型的 可控性, 利用李雅普诺夫理论构建了一个反馈控制 律来控制机器人的位姿。基于动力学模型, 他们利 用反步法构造了另外一个反馈控制律。2012 年 URAKUBO 等 ${ }^{[16]}$ 进一步展开了对该球形机器人控 制的研究, 他们提出了一种反馈控制律来控制机器 人球形外壳的角速度。2016 年, URAKUBO 等 ${ }^{[17]}$ 基于动力学模型设计了一个反馈控制器, 通过外壳 跟踪预期角速度函数来使机器人完成预期运动。他 们利用李雅普诺夫定理验证了控制律的稳定性, 并 且指出该控制器不仅可以应用到直线运动控制上, 还可以推广到圆周运动和蛇形运动上来。他们通过 试验验证了该控制器对球形机器人直线运动、圆弧 运动以及蛇形运动的控制, 图 11 展示了对该机器人 进行三种运动控制的试验结果。

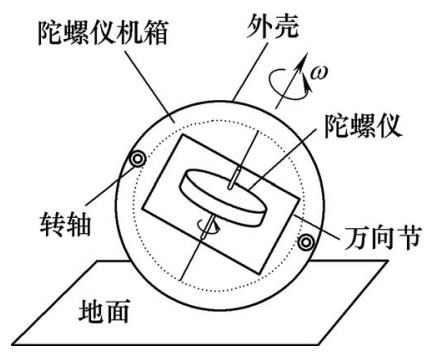

图 10 OTANI 等研究的球形机器人模型 ${ }^{[15]}$

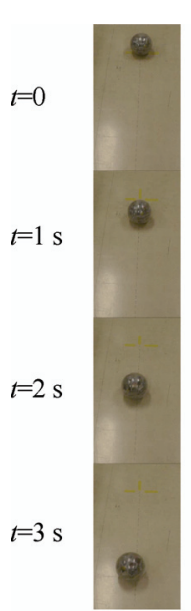

(a)

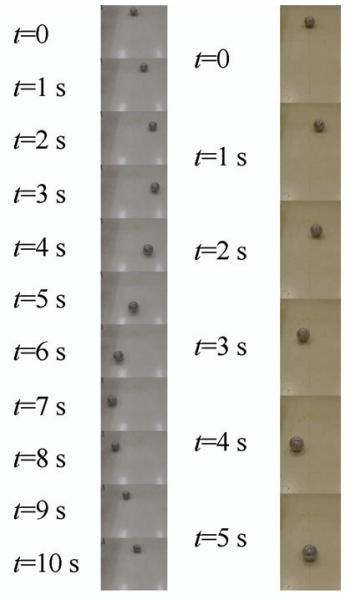

(b)

(c)
图 11 直线、圆弧及蛇形运动控制试验结果 ${ }^{[17]}$

2008 年, 瑞典的 Rotundus 公司 ${ }^{[18]}$ 利用二自由 度单摆锤驱动(图 12)研发了一款商业化球形机器人
GroundBot(图 13)。该款机器人由一个主球体和两个 小的半球体组成, 两侧小的半球形透明罩内装有摄 像头。机器人整体高 $0.6 \mathrm{~m}$, 宽 $0.8 \mathrm{~m}$, 质量 $25 \mathrm{~kg}$, 可以在多种地面运动, 如雪地、沙地、泥地等, 并 且可以漂浮在水面上。机器人通过内部的二自由度 摆锤改变整体的质心位置实现运动。当摆锤从质心 摆向前侧时, 球体将开始向前滚动, 将摆锤向左侧 或者右侧偏移将使球体向相应方向转向。该机器人 可以通过手动遥控或者通过 GPS 信号自动巡航。

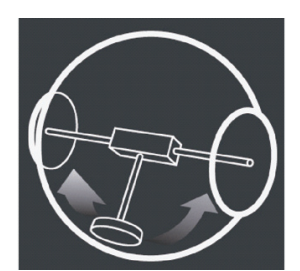

图 12 单摆球形机器人 ${ }^{[18]}$

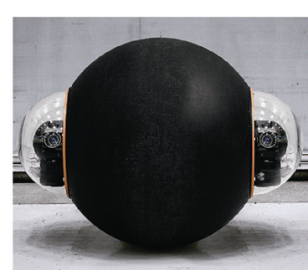

图 13 GroundBot 机器人 ${ }^{[18]}$
研究对象同样为单摆锤驱动的球形机器人, 2012 年, 比利时鲁汶大学 (KU Leuven) 的 KAYACAN ${ }^{[19]}$ 等对机器人建立了运动学模型, 利用 Euler-Lagrange 方程建立了动力学模型。他们将非完 整的强非线性运动方程解耦合为两个较简单的子系 统, 然后通过反馈线性化和比例 - 微分 (Proportion-derivative, PD) 模糊控制器实现了对机器 人运动的闭环控制, 并且通过直线轨迹运动和圆形 轨迹运动的仿真验证了该控制器。在此基础上, 2013 年 KAYACAN 等 ${ }^{[20]}$ 又提出了一种模糊神经网络 (Fuzzy neural network, FNN)控制器, 利用在线学习 算法较好解决了机器人因动力学建模不准确而影响 控制精确度的问题。与传统的 PD 控制器相比, 该 速度控制器在稳态误差以及瞬态响应方面都具有优 势。其中 PD+FNN 控制器与传统 PD 控制器对误差 处理的差别可以从图 14 中看出。

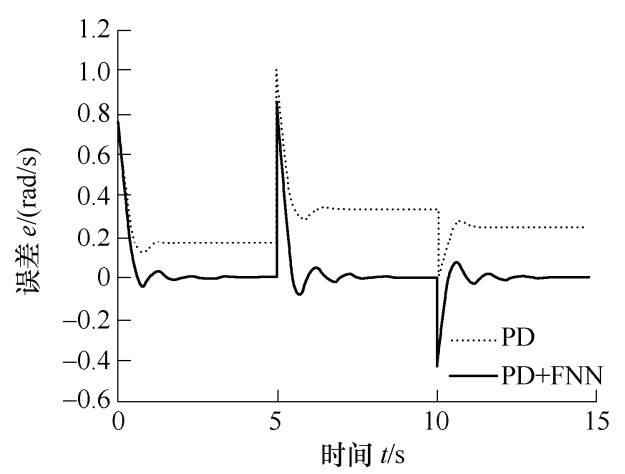

图 14 PD+FNN 控制器与 PD 控制器对比图 ${ }^{[20]}$

针对单摆锤驱动球形机器人, 2014 年, 伊朗 德黑兰大学(University of Tehran)的 ROOZEGAR 等 ${ }^{[21-22]}$ 利用 BELLMAN ${ }^{[23]}$ 提出的动态规划(Dynamic Programming, DP) 算法实现了在平面上的路径规 
划及控制, 利用 DP 算法的优点是: 无论机器人当 前状态是什么, 它都可以根据目标位置生成闭环控 制输入给机器人。他们提出的路径规划算法在有障 碍物的情况下仍然适用, 并通过试验验证了提出的 路径规划及控制算法。图 15 展示的是在有障碍物的 情况下, 规划路径与试验所得路径之间的对比。2016 年, ROOZEGAR 等 ${ }^{[24]}$ 运用强化学习 (Reinforcement learning, RL)算法提出了另外一种路径规划算法, 通 过仿真证明了该算法可以规划出从任意位置出发到 达预定目标点的优化路径。2017 年, ROOZEGAR 等 ${ }^{[24]}$ 继续研究了其在坡面上的建模和运动控制问 题, 他们将球体和斜坡简化为二维模型, 只考虑机 器人的上坡和下坡运动, 利用拉格朗日方程建立了 系统的动力学模型, 利用比例 - 积分 - 微分 (Proportion-integral-derivative, PID)控制器实现了球 形机器人在坡面上的稳定控制, 利用模糊控制器和 PID 控制器完成了机器人在坡面上的上坡运动、下 坡运动以及定点控制。最后, 他们通过仿真和试验 的方式验证了该控制器。图 16 为该机器人在 $20^{\circ}$ 斜 坡上进行定点控制的仿真和试验结果。

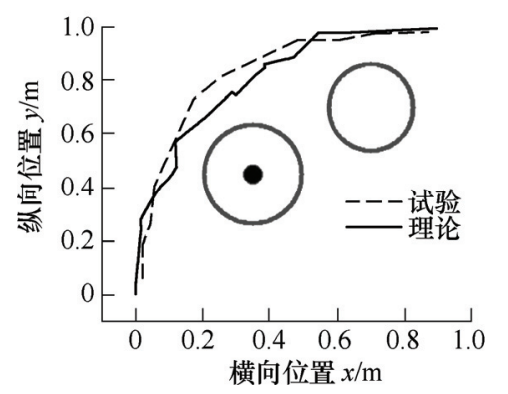

图 15 有障碍物的路径规划及控制试验结果 ${ }^{[22]}$

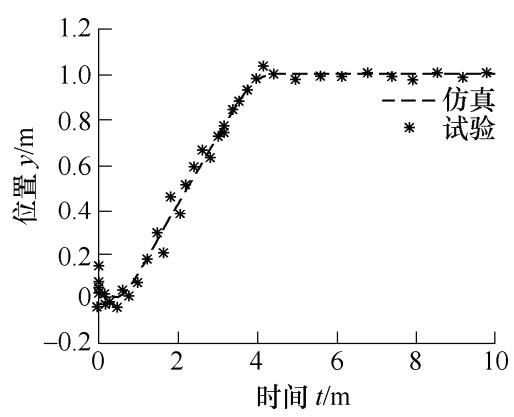

图 16 定点控制仿真和试验结果 ${ }^{[25]}$

针对单摆锤驱动球形机器人, 2016 年, 印度理 工学院孟买校区 (Indian Institute of Technology Bombay)的 GAJBHIYE 等 ${ }^{[26]}$ 建立了非完整约束 Euler-Poincaré 方程, 并且利用李括号理论证明了该 机器人是局部位形可控的 ${ }^{[27-28]}$, 即机器人的位姿可 以通过支架和摆锤在短时间内得到控制。

2012 年, 日本香川大学(Kagawa University)的 LIN 等 ${ }^{[29]}$ 研制了一款水下球形机器人(图 17), 该机
器人由三个矢量推进器驱动, 可以完成前行、上浮 以及旋转等基本运动。2 015 年, $\mathrm{LI}$ 等 ${ }^{[30]}$ 针对三推进 器机器人能耗大的问题提出了一种优化设计, 新的 水下球形机器人(图 18) 由四个垂直对称排布的矢量 推进器驱动。LI 等利用 Lagrange-Routh 方程为机器 人建立了动力学模型, 应用有限元法通过 CATIA 软件建立模型, 并导入 ADAMS 软件进行仿真, 仿 真结果验证了模型的正确性和机器人的路径跟随 性能。

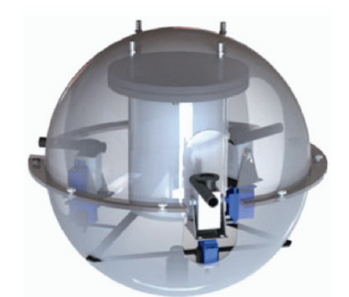

图 17 LIN 等的机器人 ${ }^{[29]}$

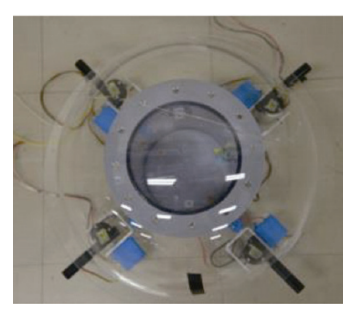

图 18 LI 等的机器人 ${ }^{[30]}$
2013 年，伊朗 University of Tabriz 的 MAHBOUBI 等 ${ }^{[31]}$ 研制了一款双摆锤驱动的球形机 器人。其结构如图 19 所示, 两个摆锤 $(2 、 3)$ 由两个 独立电动机(4、5)驱动, 摆锤通过线性电动机或滚 珠丝杜(6、7)与驱动电动机 $(4 、 5)$ 相连, 所以摆锤在 球壳内的位置可以通过 6 和 7 改变。 8 和 9 是两侧 独立驱动单元的控制器。该机器人可以实现直线运 动、圆弧运动、原地转向以及跳动。当两侧摆锤位 置对称、电动机驱动两个摆锤绕同一方向等速转动 时, 机器人将沿直线运动; 如果摆锤位置不对称或 两侧电动机转速不同, 机器人将沿圆弧转动。当两 侧摆锤沿相反方向低速转动时可以实现机器人的原 地转向, 当两侧摆锤高速转动时机器人跳动。他们 利用 Boltzmann-Hamel 方程为机器人建立了动力学 模型, 并且对直线运动、圆周运动和跳动分别展开 动力学分析, 实现了对机器人的开环控制。

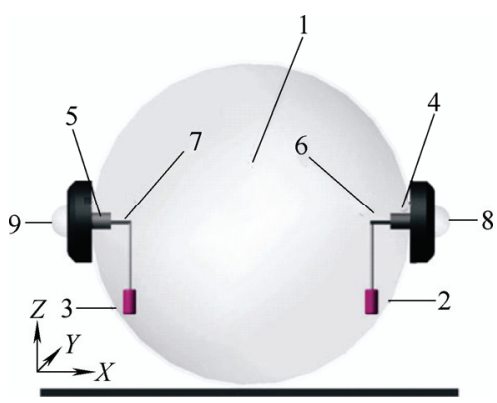

图 19 MAHBOUBI 等研制的球形机器人结构图 ${ }^{[31]}$

2015 年, 加拿大麦吉尔大学(McGill University) 的 HOGAN 等 ${ }^{[32]}$ 针对球形机器人在任意曲面上的运 动建模问题展开了研究。球形机器人模型如图 20 所示, 该机器人靠外力 (例如风力)驱动, 机器人靠 
滚动发电并存储来给机载设备供电。他们用四元数 表示机器人的姿态, 利用拉格朗日方程建立起机器 人系统的运动方程, 并通过仿真加以验证。图 21 所示的是该机器人从坡上滚下, 然后通过起伏路面 的仿真结果。

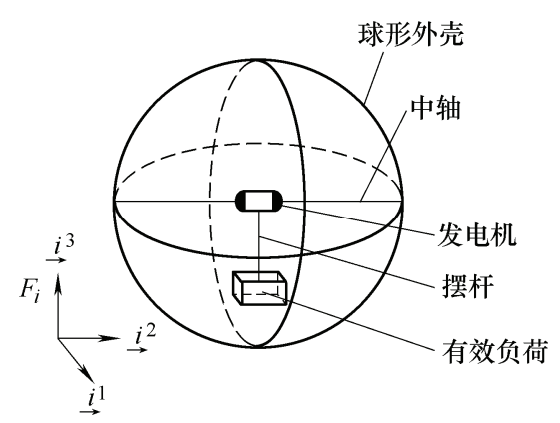

图 20 HOGAN 等研究的球形机器人结构图 ${ }^{[32]}$

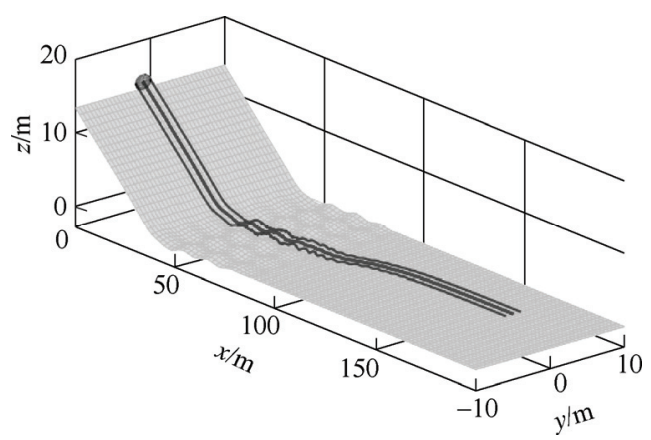

图 21 机器人运动仿真结果 ${ }^{[32]}$

2017 年, 美国中央密执安大学(Central Michigan University)的 DEJONG 等 ${ }^{[33]}$ 设计并制作了一款四摆 锤驱动的球形机器人(图 22), 四个摆锤以四面体形 式排布。DEJONG 等对该机器人建立了动力学模型, 通过开环控制仿真验证了动力学模型的正确性。

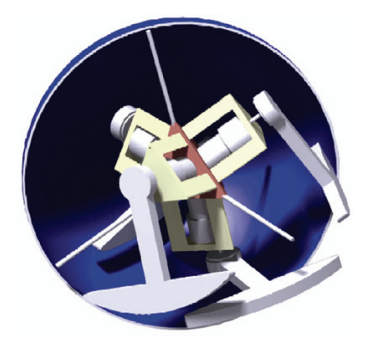

图 22 控制单个摆锤的仿真试验结果 ${ }^{[33]}$

\section{2 国内研究进展}

\subsection{BHQ 系列球形机器人}

北京航空航天大学机器人研究所复杂机构与智 能控制实验室 $(\mathrm{CMIC})^{[34]}$ 自 2000 年在国内率先开展 球形机器人的研究, 运用不同驱动方式先后研制了 多种不同结构的球形机器人, 其中包括 BHQ-1、 BHQ-2、BHQ-3、BHQ-4 和 BHQ-5。这些球形机器
人多采用全封闭结构, 运动灵活, 结构精巧, 具有 在复杂环境中开展探测活动的能力。

2001 年, 北京航空航天大学的战强等 ${ }^{[35]}$ 研制了 一种通过控制质量块位置来实现机器人运动的球形 探测机器人 BHQ-1(图 23)。2005 年, 战强等 ${ }^{[36-37]}$ 运用拉格朗日方程对 BHQ-1 机器人建立了动力学 模型, 通过对机器人运动性能的分析优化了 BHQ-1 的设计, 从而制作了新的球形机器人样机, 该机器 人在机构设计和电动机布局上更合理, 便于电动机 的安放, 而且具有更小的转弯半径。电动机 1 通过 传动装置与球壳连接, 直接驱动球壳的前进与后退。 电动机 2 控制重块左右偏摆来实现转弯运动。

2006 年, 战强等 ${ }^{[38]}$ 对球形机器人的电动机控制 进行了研究, 建立了直流电动机的数学模型, 设计 了速度、电流双闭环电动机控制系统, 并通过 MATLAB 确定了 BHQ-1 电动机控制系统的 PID 参 数。机器人可以通过红外传感器身避障碍物, 避障 试验验证了电动机控制系统的正确性。同时, 战强 等 ${ }^{[39]}$ 对 BHQ-1 的动态轨迹规划问题进行了研究, 利 用 Boltzmann-Hamel 方程建立 BHQ-1 的动力学模 型, 基于模型对直线运动和圆周运动两种情况下两 个电动机的驱动力矩进行规划设计。2008 年, 陈明 等 ${ }^{[40]}$ 对 BHQ-1 建立了运动学模型, 并且通过准速度 (quasi-velocity)对方程进行了简化。基于运动学模 型, 他们利用 Hamilton 方程对 BHQ-1 进行了基于 最短时间和最低能耗的轨迹规划, 该方法同样通过 避障试验进行了验证。

2008 年, 刘增波等 ${ }^{[41]}$ 对球形机器人 BHQ-2(图 24)的运动控制展开了研究。他们基于旋量理论解决 了 BHQ-2 的速度雅可比矩阵推导问题以及速度级 运动学逆问题, 利用李代数验证了系统的可控性, 基于速度逆解对机器人实施了轨迹跟踪开环控制。

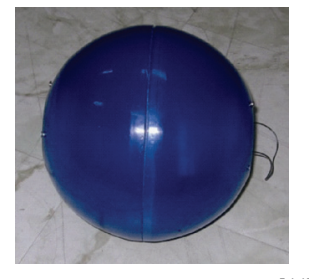

图 23 BHQ-1 机器人 ${ }^{[44]}$

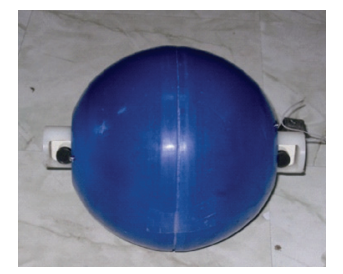

图 24 BHQ-2 机器人 ${ }^{[41]}$
战强等 ${ }^{[42]}$ 基于运动学模型运用 Gauss-Newton 算法对 BHQ-2 进行了轨迹规划研究。他们指出, 要 想获得较高的控制精度, 必须实现闭环控制。战强 等 ${ }^{[43]}$ 在 2008 年运用 Boltzmann-Hamel 方程对 BHQ-2 建立了动力学模型, 运用反步法设计了一个 轨迹跟踪控制器, 其收玫性通过李雅普诺夫方程进 行验证, 首次实现了对机器人两个位置状态量和一 
个姿态角的轨迹跟踪控制, 并通过跟踪直线轨迹和 圆形轨迹的仿真证明了该闭环控制器的有效性和准 确性, 圆形轨迹跟踪仿真结果如图 25、26 所示。

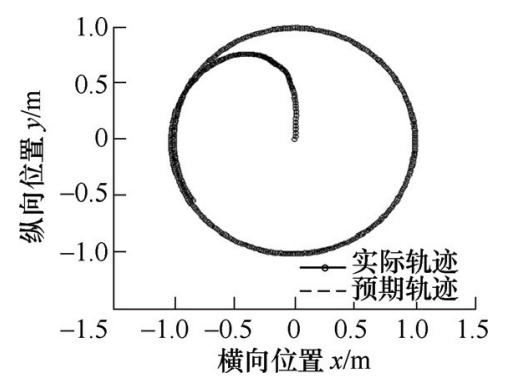

图 25 圆形轨迹跟踪仿真结果 ${ }^{[43]}$

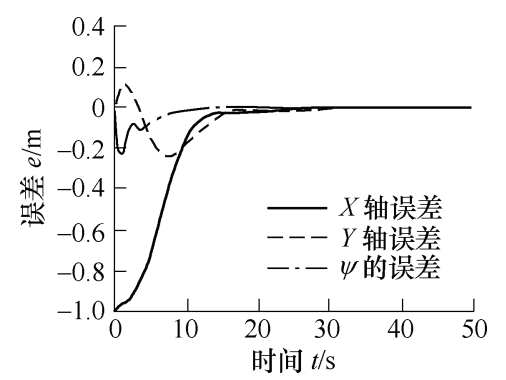

图 26 三个状态量的跟踪误差曲线 ${ }^{[43]}$

2011 年, 蔡尧等 ${ }^{[44]}$ 通过旋量理论建立了 BHQ-1 球形机器人的运动学模型, 利用李代数证明了该系 统的可控性, 并基于反向传播(Back propagation, BP) 神经网络提出了一种求逆运动学模型的方法。2012 年, 蔡尧等 ${ }^{[45]}$ 基于 BHQ-1 球形机器人的运动学模 型, 运用神经动力学 Shunting 模型构造了对机器人 位置状态参数的反馈控制律, 并且利用李雅普诺夫 稳定性原理证明了其稳定性, 实现了对直线轨迹、 圆形轨迹以及正弦函数轨迹的跟踪控制, 图 27 为正 弦函数轨迹的仿真结果。动力学方面, 蔡尧等 ${ }^{[46]}$ 运 用 Euler-Lagrange 方程建立了 BHQ-1 直线运动的动 力学模型, 运用小脑模型关节控制器 (Cerebellar model articulation controller with general basis function, CMAC_GBF)神经网络和信用分配(Credit assignment, CA)学习方法提出了一种控制器, 仿真 验证了该控制器的快速收敛性。战强等 ${ }^{[47]}$ 利用 PID 控制器同样实现了对机器人直线运动的位置和转角 的控制。基于 BHQ-1 平面运动的动力学模型, 蔡尧

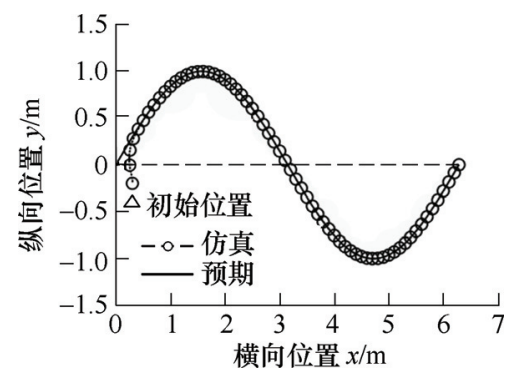

图 27 正弦函数轨迹跟踪仿真结果 ${ }^{[45]}$
等 ${ }^{[48]}$ 提出了一种基于拟人 Guide-Tracker 模式的模 糊控制策略, 它模拟了人的跟踪行为, 可以在实现 路径跟踪的同时保持稳定的侧倾角。其“U”形路径 仿真结果如图 28 所示, 其中图 28 下方的图为侧倾 角的变化。
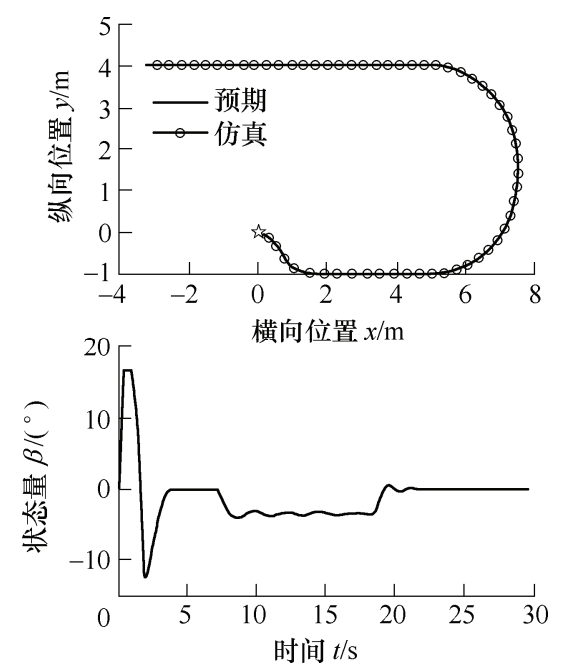

图 28 模糊 Guide-Tracker 跟踪算法仿真结果 ${ }^{[48]}$

2011 年, 郑明慧等 ${ }^{[49]}$ 融合 BHQ-1 的运动学模 型和动力学模型, 设计了对该球形机器人的路径跟 随控制器。该控制器首先通过运动学模型计算预期 速度, 然后通过动力学模型将其转化为实际力矩, 并通过圆形路径、直线路径等的仿真结果验证了该 控制器的有效性。图 29 和图 30 展示了从圆形路径 转换到直线路径的复合路径跟随控制的仿真结果。 2012 年, 郑明慧等 ${ }^{[50}$ 运用 PD 控制器和径向基函数 (Radial basis function, RBF)神经网络基于 BHQ-1 的 运动学模型和不准确的动力学模型设计了一个对机

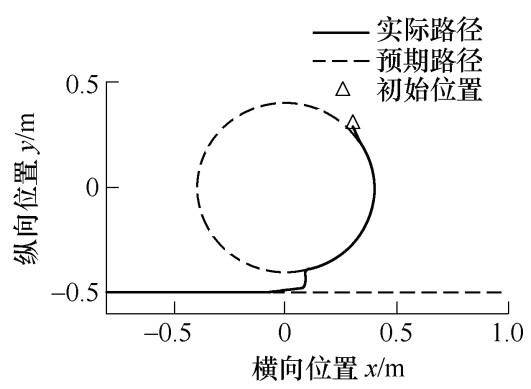

图 29 路径跟随控制仿真结果 ${ }^{[49]}$

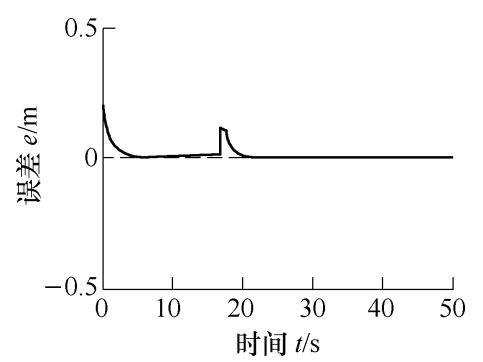

图 30 仿真跟随误差 ${ }^{[49]}$ 
器人位置的轨迹跟踪控制系统, 通过调节 RBF 神经 网络的权重来减少跟踪误差, 该控制系统有效解决 了动力学模型的不准确性和外部扰动带来的问题。 该控制系统的稳定性通过李雅普诺夫理论得到证 明, 并且通过仿真的方式加以验证。

2011 年, 战强等 ${ }^{[51]}$ 进一步研发了 BHQ-3 球形 机器人。其内部装有一个平台, 上部装有一个转向 驱动小轮以及转向电动机, 下部装有一个大轮以及 直行驱动电动机, 电动机 1 带动大轮转动以驱动球 体前行, 电动机 2 带动小轮转动从而实现球体的转 向。他们对 BHQ-3 进行了直线运动、转向运动、爬 坡运动以及越障运动的运动性能分析, 建立了运动 学模型并证明了其可控性。他们对 BHQ-3 进行了开 环控制仿真, 并对 BHQ-3 机器人样机进行了一系列 试验, 试验中机器人表现出了良好的运动性能。 BHQ-3 可以爬上 $17^{\circ}$ 的斜坡, 可以越过高度不超过 $30 \mathrm{~mm}$ 的障碍物, 并且可以适应不同环境, 例如沙 地和水面(图 31)。
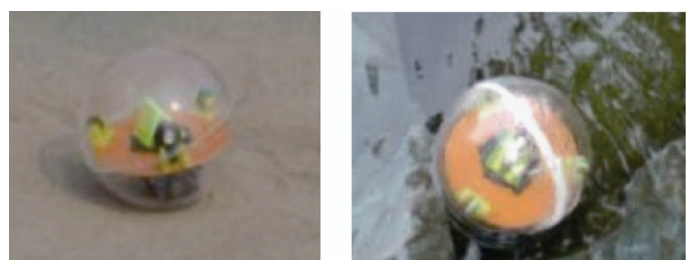

图 31 沙地及水面上的 BHQ-3 球形机器人 ${ }^{[51]}$

2014 年, 战强等 ${ }^{[52-53]}$ 研发了一款用于野外环境 的可变形球形探测机器人 BHQ-5(图 32), 该机器人 越野能力强, 能够适应野外的草地、沙地、斜坡面、 垂直障碍等复杂地形。在存储和运输时, 该机器人 的形态就是一个闭合的球。当需要执行任务时, 球 形机器人就展开变形为一个轮爪式的机器人。机器 人由三部分组成, 第一部分是左右对称的球壳、轮 爪等组件; 第二部分是机器人的支撑尾轮, 对机器 人起支撑作用, 并且可以平衡电动机力矩; 第三部 分是中央平台, 包括控制板、电动机等部件, 是整 个机器人的安装基准。该机器人由两个电动机分别 驱动两侧球壳的转动。当两个电动机同向同速转动 时机器人将直线行驶, 即前行或后退。当两个电动
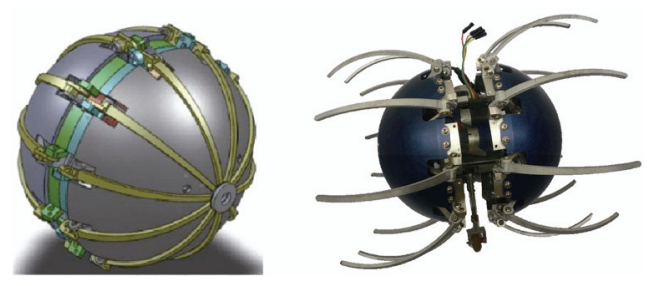

图 32 BHQ-5 收缩状态(左)与完全展开状态(右)
机差速转动时, 机器人将完成转弯运动。另外, 当 两个电动机以相同的转速沿相反方向转动时, 机器 人将实现原地转弯。他们利用 ADAMS 软件对机器 人模型进行了直线运动、越障等运动的仿真分析, 最后利用样机进行了野外的实地测试, 仿真和试验 的结果验证了该机器人的良好运动性能。

\section{2 国内其他球形机器人}

2004 年, 北京邮电大学的肖爱平等 ${ }^{[54]}$ 设计一款 球形机器人 BYQ-II, 该机器人由两个电动机驱动, 一个通过改变重块质心位置驱动机器人前行, 另一 个基于角动量守恒原理驱动机器人实现原地转向。 2005 年, 孙汉旭等 ${ }^{[55]}$ 设计了另一款二驱动球形机器 人 BYQ-III(图 33), 两个电动机可以驱动一个二自 由度重块绕两个相互垂直的轴转动。2007 年, 王亮 清等 ${ }^{[56-57]}$ 进一步分析了 BYQ-III 的圆周运动性能以 及爬坡和弹跳能力。2008 年, 刘大亮等 ${ }^{[58]}$ 在研究球 形机器人 BYQ-III 的动力学控制时提出了一种低速 旋转时的简化方法: 球形机器人的直线运动和转弯 运动之间存在的加速度耦合可以去耦并且两平面上 的运动方程是相同的, 由于输入为两个正交的力矩, 通过输入变换将动力学模型转换为一个两输入的二 阶系统。然后提出了侧倾运动的全状态反馈控制器 和前向运动的 PI 控制器, 保证被控的运动状态收 敛。 2009 年, 孙汉旭等 ${ }^{[59]}$ 基于凯恩方程为 BYQ-III 球形机器人建立了完整动力学模型, 并通过开环运 动控制试验验证了该动力学模型。

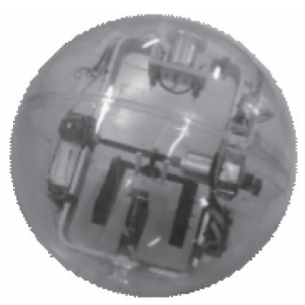

图 33 BYQ-III 球形机器人样机 ${ }^{[58]}$

2007 年, 西安电子科技大学的李团结等 ${ }^{[60]}$ 设计 了一款与 JAVADI 等设计的球形机器人(图 6) 类似的 机器人, 不同的是李团结等为机器人设计了一个位 于中心的稳定平台。李团结等 ${ }^{[61]}$ 利用 LagrangeRouth 方程建立了机器人的动力学模型, 消去未知 拉格朗日乘子后得到了完整动力学模型。

2007 年, 哈尔滨工业大学的岳明等 ${ }^{[62]}$ 针对单 摆驱动球形机器人前向滚动状态下内部构件的稳 定控制问题展开研究。由于只考虑前向滚动, 机 器人被简化为一个二维平面上滚动的圆盘。他们 利用基于能量耗散形式下的拉格朗日方程建立其 
动力学模型, 然后通过线性化和仿射变换对模型 进行简化, 并构建了状态观测器对系统中某些不 可测量的状态变量进行实时观测, 设计出了系统 的状态反馈控制器。2014 年, 岳明等 ${ }^{[63]}$ 基于扩展 状态观测器, 提出了一种自适应分层滑模控制器 以实现单摆驱动球形机器人的前向运动。该控制 系统具有鲁棒性和自适应性能。图 34、35 展示了 机器人沿预期力矩轨迹进行跟踪控制时得到的仿 真数据, 其中图 34 为力矩的跟踪曲线, 图 35 为 四个状态量(球壳转角及转速、摆锤抬角及相应转 速)的跟踪误差曲线。

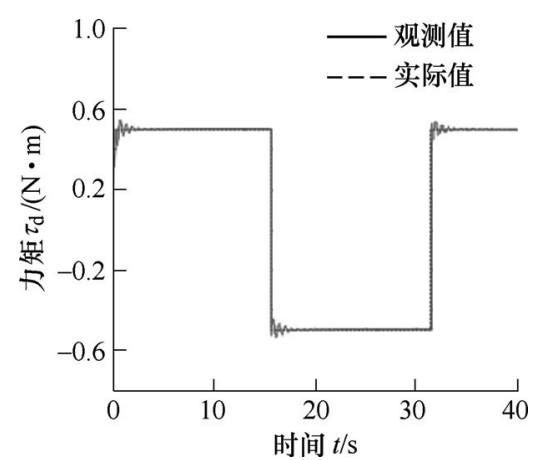

图 34 力矩跟踪曲线 ${ }^{[63]}$
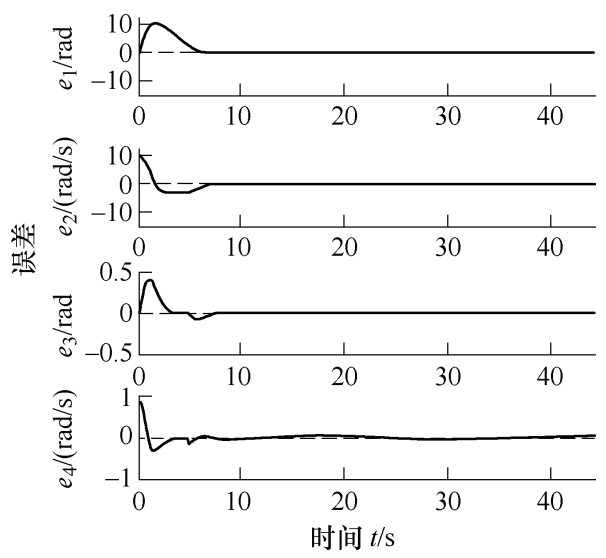

图 35 状态量跟踪误差曲线 ${ }^{[63]}$

2009 年哈尔滨工业大学的赵勃等 ${ }^{[64]}$ 设计了一 款双偏心质量块驱动球形机器人(图 36)。该机器人 采用两个对称的驱动单元分别驱动一个偏心质量 块, 与二自由度单摆驱动球形机器人相比, 该机器 人可以提供更充足的偏心力矩。另外, 该机器人外 壳采用了椭球形, 这样可以在一定程度上避免球体 的侧翻。针对该机器人的直线运动, 赵勃等利用拉 格朗日方程推导了其动力学方程, 并提出了一种基 于高斯函数的控制方法, 使机器人直线运动时可以 稳定启停且在中间的匀速阶段速度可控。另外, 他 们设计了对质量块摆角的反馈控制器来实现对运动 速度的跟踪控制。他们通过仿真和样机试验验证了 控制器的有效性, 图 37 展示的是速度跟踪控制仿真
的结果。同样利用双摆锤驱动, 2017 年, 天津大学 的杨伟 ${ }^{[65]}$ 等设计了一款球形机器人, 对机器人的爬 坡、转向和弹跳进行了动力学分析。

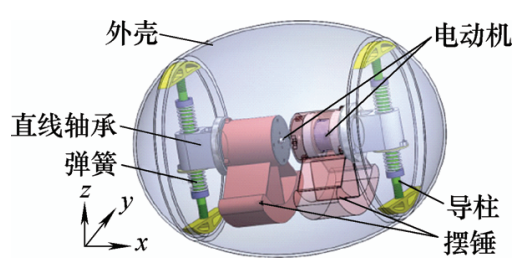

图 36 赵勃等研究的球形机器人模型 ${ }^{[64]}$

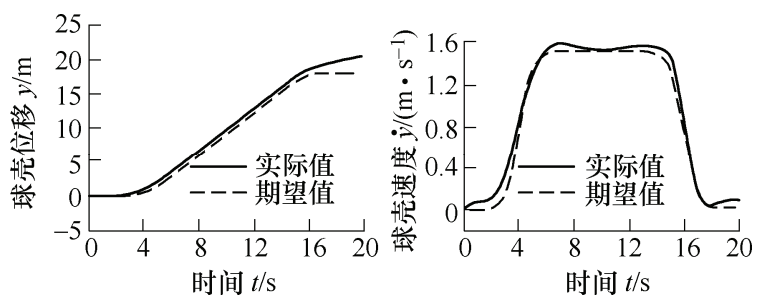

图 37 球壳位移和速度试验曲线 ${ }^{[64]}$

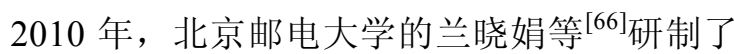
一款六自由度的水下球形机器人 BYSQ-2(图 38), 该机器人通过内置机构调节姿态, 推进运动仅由 一个推进器实现。该机器人内部装有一个中心导 管, 导管内部装有一个推进器, 导管外部与机器 人耐压舱相连。耐压舱内装有两个电动机, 通过 转动配重块调整导管口的方向, 然后启动推进器 推动机器人运动。他们对机器人进行了动力学分 析, 基于流体动力学建立了控制球壳姿态的模型, 对模型近似线性化后用模糊自适应 PID 方法控制 配重的摆角以保持球壳姿态的稳定。2016 年, 北 京邮电大学的李艳生等 ${ }^{[67]}$ 深入研究了 BYSQ-2 的 水底滚动特性, 在滚动方程的基础上, 通过仿真 和试验分析了机器人的质量和电动机转速对机器 人在水底滚动的影响。

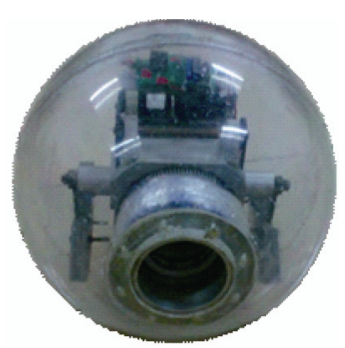

图 38 BYSQ-2 水下球形机器人 ${ }^{[66]}$

2013 年, 中国台湾大学的 $\mathrm{CHEN}$ 等 ${ }^{[68]}$ 研制了 一款球形机器人(图 39), 由两个正交排布的电动机 驱动, 电动机驱动滚轮转动, 滚轮带动内部驱动小 球滚动, 通过摩擦带动外部球壳滚动。他们通过仿 真分析机器人的运动性能, 优化各项参数后制作样 机，用试验验证了该机器人的全向运动性能。 


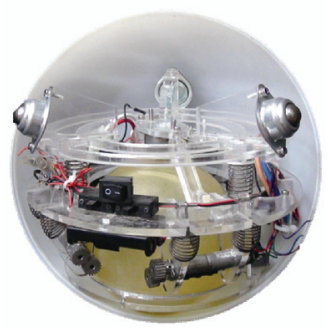

图 39 CHEN 等研究的球形机器人样机 ${ }^{[68]}$

2013 年, 北京邮电大学的叶平等 ${ }^{[69-70]}$ 设计了一 款具有立体视觉的球形机器人 Visionbot, 机器人靠 单摆锤驱动。立体视觉提高了球形机器人的环境感 知能力, 运动控制精度得到了相应提高。他们通过 拉格朗日方程建立了该球形机器人的动力学模型, 并且通过合理简化减少了模型的状态变量。他们利 用模型解耦、线性化以及状态反馈等方法建立了该 球形机器人的内环力矩控制器和外环路径跟踪控制 器, 并且采用延时环节将视觉反馈添加到运动控制 模型中。他们通过运动仿真试验研究了重摆质量和 视觉反馈延时对机器人运动控制性能的影响(图 40), 同时也验证了控制系统的正确性。

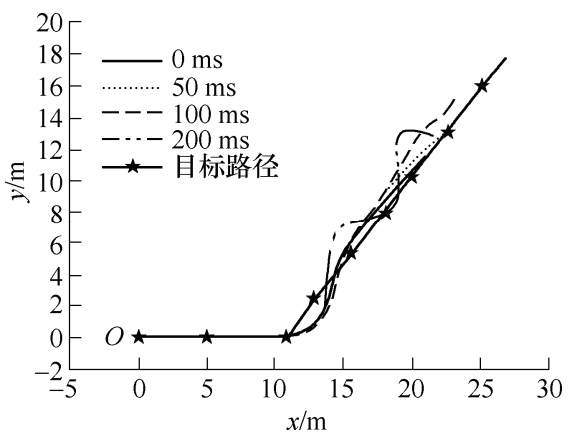

图 40 不同视觉反馈延时下机器人的运动路径 ${ }^{[69]}$

\section{3 研究进展及发展趋势的分析总结}

\section{1 球形机器人的分类}

球形机器人的运动原理主要有两种: 一种是通 过质心偏移, 机器人在重力矩的作用下运动; 另一 种是保证机器人的质心永远位于地面接触点的正上 方, 从而在接触点处的重力矩为零, 机器人基于角 动量守恒原理运动。除了这两种基本的运动原理以 外, 还有水下球形机器人、基于形变原理运动的其 他球形机器人, 如图 41 所示。

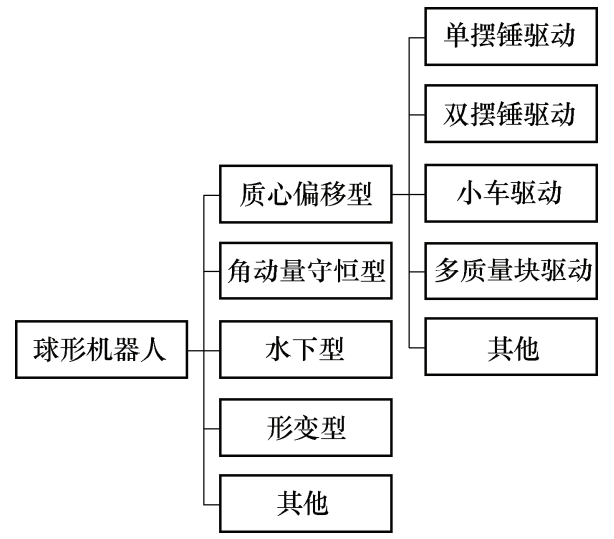

图 41 球形机器人分类

\subsection{1 质心偏移型}

目前, 大多数球形机器人的运动原理是质心偏 移, 因为质心偏移型球形机器人的运动形式更为直 观、更为明朗。对于质心偏移型球形机器人, 按照 其内部结构分类主要有四种形式: 单摆锤驱动、双 摆锤驱动、小车驱动以及多质量块驱动(表 1)。

表 1 各类质心偏移型球形机器人的比较

\begin{tabular}{|c|c|c|c|c|}
\hline 驱动方式 & 单摆锤驱动 & 双摆锤驱动 & 小车驱动 & 多质量块驱动 \\
\hline 示意简图 & & & & \\
\hline 控制输入数量 & 2 & $2 \sim 4$ & $2 \sim 3$ & $3 \sim 4$ \\
\hline 样例 & $\begin{array}{l}\text { BHQ-1, BHQ-2, GroundBot, } \\
\text { Visionbot }\end{array}$ & $\begin{array}{l}\text { 赵勃等, MAHBOUBI 等 } \\
\text { 的研究对象 }\end{array}$ & BHQ-3, Sphericle, SPRK+ & $\begin{array}{l}\text { AUGUSTt, 李团结等 } \\
\text { 的研究对象 }\end{array}$ \\
\hline 优点 & $\begin{array}{l}\text { 结构简单明朗, 驱动方式直 } \\
\text { 观, 适合平面运动 }\end{array}$ & $\begin{array}{l}\text { 爬坡能力改善、转弯灵活、可 } \\
\text { 以弹跳 }\end{array}$ & $\begin{array}{l}\text { 爬破及越障能力强, 内部平台 } \\
\text { 易于设备的搭载 }\end{array}$ & 越障能力强 \\
\hline 缺点 & 越障能力较弱, 转弯不灵活 & 直线运动较困难 & $\begin{array}{l}\text { 车轮与球壳内壁间存在非完 } \\
\text { 整约束 }\end{array}$ & $\begin{array}{l}\text { 轨迹规划及控制复杂, 难以搭 } \\
\text { 建稳定平台 }\end{array}$ \\
\hline 目前研究进展 & $\begin{array}{l}\text { 研究最多, 可以建立运动学模 } \\
\text { 型和动力学模型, 实现了对直 } \\
\text { 线、圆形以及爬坡运动的闭环 } \\
\text { 控制 }\end{array}$ & $\begin{array}{l}\text { 研究较少, 可以建立动力学模 } \\
\text { 型, 实现了对平面直线运动的 } \\
\text { 闭环控制 }\end{array}$ & $\begin{array}{l}\text { 研究较多, 可以建立运动学模 } \\
\text { 型和动力学模型, 实现了闭环 } \\
\text { 运动控制 }\end{array}$ & $\begin{array}{l}\text { 研究较少, 可以建立动力学模 } \\
\text { 型并对其进行开环控制 }\end{array}$ \\
\hline
\end{tabular}


针对单摆锤驱动球形机器人的研究最为丰富, 例如战强等研制的 BHQ-1 和 BHQ-2 机器人、瑞典 Rotundus 公司研发的 GroundBot 机器人、叶平等研 究的 Visionbot 机器人等。该类机器人一般由两个电 动机驱动一个二自由度摆锤, 摆锤向前或向后抬起 将驱动机器人前后滚动, 摆锤侧偏时机器人将完成 转向运动, 侧偏幅度越大, 转弯半径越小。该类机 器人的结构简洁明朗, 驱动控制方式直接, 但是因 其结构特点导致越障能力较差, 一般无法实现原地 转向, 因此该类机器人适合在平坦的地面上工作。

第二类质心偏移型机器人靠双摆锤驱动, 赵勃 等以及 MAHBOUBI 等研究的机器人均采用双摆锤 驱动。该类机器人通过两侧的两个独立摆锤来改变 机器人整体的质心位置, 两个独立摆锤决定了该类 机器人至少由两个电动机驱动, 例如赵勃等研究的 机器人的电动机数量为二, 两个电动机分别驱动一 个摆锤转动; 再例如 MAHBOUBI 等研究的球形机 器人, 它的电动机数量为四, 每个摆锤由两个电动 机控制, 一个控制摆锤绕轴转动, 另一个控制摆锤 沿轴向平移。当两侧摆锤位置对称、电动机同向等 速转动时, 机器人可以完成直线运动; 当两侧电动 机差速转动时机器人将转向, 改变两侧摆锤位置的 对称性可以辅助机器人转向。另外, 当两侧电动机 以相反方向快速转动时, 机器人将完成弹跳运动; 当转速很低时, 机器人可以完成原地转向。该类机 器人与单摆锤驱动球形机器人相比, 驱动力矩更强 因此爬坡能力改善, 可以实现跳跃和原地转向, 但 是由于直线运动时需要严格保持两侧摆锤的对称 性, 因此该类机器人完成直线运动较为困难。

第三类质心偏移型机器人通过内部小车驱动。 HAMEL 等、战强等采用的是独轮小车, BICCHI 等及美国 Sphero 公司采用的是双轮驱动小车。这类 机器人的电动机数量一般为二, 可以两个电动机输 出轴正交分布, 一个控制转向另一个控制前行, 例 如 BHQ-3; 也可以两个电动机驱动两个平行排布的 独轮, 两个轮子同向等速转动时直行, 差速转动时 转向, 例如 SPRK + 。该类机器人爬坡及越障能力较 强, 并且内部小车平台空间大, 易于搭载传感器等 设备。但是内部车轮与球壳内壁之间存在非完整约 束, 增加了对机器人进行建模和控制的复杂度。

第四类质心偏移型机器人通过改变多个质量块 的位置运动, 李团结等、JAVADI 等以及 MUKHERJEE 等研究的球形机器人采用了这种驱 动方式。该类机器人内部安装有三个或四个固定轴, 每个轴上安装有一个质量块, 分别由一个电动机驱 动滑块运动, 因此该类机器人的电动机数量为三个
或四个。JAVADI 等和李团结等的机器人内部均安 装有四个固定轴, 四个轴的排布类似于甲烷分子的 空间正四面体结构。MUKHERJEE 等制作了一个安 装有三个固定轴的球形机器人。该类机器人的越障 能力强, 机器人被障碍物卡住时容易调整重心翻过 障碍, 但是由于该类机器人的驱动方式不直观, 对 机器人进行轨迹规划和控制较为困难, 并且难以在 内部搭建较大空间的稳定平台。除了这四类主要的 质心偏移型驱动方式以外, 还存在少量其他形式的 驱动方式, 例如 DEJONG 等设计的四摆锤驱动的球 形机器人。

\section{1 .2 角动量守恒型}

该类机器人需要其保证质心严格位于球心处, 这样机器人对地面接触点处的重力矩为零, 机器人 不会自己侧倾。机器人内部安装有一个或多个转子, 电动机驱动转子转动, 基于角动量守恒原理, 机器 人球壳会受到相反方向等大的力矩，在此力矩作用 下球形机器人将在地面滚动。JOSHI 等设计的球形 机器人由两个电动机驱动, 两个转子垂直排布, MURALIDHARAN 等研究的球形机器人由三个电 动机驱动, 三个转子正交排布。OTANI 等研制的角 动量守恒型球形机器人由三个电动机驱动, 其中一 个电动机驱动内部陀螺的持续高速转动, 通过另外 两个电动机改变陀螺转轴, 在陀螺效应下机器人外 壳将受到驱动力矩而滚动。

\section{1 .3 水下型}

水下球形机器人可以分为两类, 一类是利用与 陆上球形机器人类似的原理, 通过质心偏移调整机 器人的姿态, 然后在推进器的作用下运动, 例如兰 晓娟等设计的 BYSQ-3; 另一类水下球形机器人只 是因为其外壳为球形，基于球形外壳排布多个推进 器, 运动原理与普通的水下机器人一样, 例如 LIN 等设计的机器人。

\subsection{4 形变型}

与一般球形机器人的刚性结构不同, 该类球形 机器人为软体结构, 通过改变外形来推动机器人运 动。例如, SUGIYAMA 等研制的利用记忆合金形变 的球形机器人和 WAIT 等研究的利用充放气产生形 变的球形机器人。对该类球形机器人进行精确控制 较为复杂。

\section{1 .5 其他类型}

除了上面提到的四种类型的球形机器人, 目前 还存在着少量的其他类型的球形机器人。例如战强 等研制的 BHQ-5 球形机器人, 该机器人左右两侧球 壳分别由一个电动机驱动, 两侧球壳靠中央平台连 接, 该球形机器人的运动原理与独立驱动的并排二 
轮小车类似。再例如 HOGAN 等研究的靠外力驱 动的球形机器人, 该机器人在风力等外力的作用 下运动。

\section{2 球形机器人的建模与控制}

目前, 球形机器人的结构设计大同小异, 球形 机器人的研究重点已经转移至控制方面。在球形机 器人出现的初期, 研究重点是机器人结构的设计和 样机的开发, 通过最简单的开环控制研究机器人的 运动性能。后来对机器人控制的要求越来越高, 例 如控制精度、控制姿态等。对机器人进行控制, 首 先要针对机器人的结构特性建立模型, 模型可以分 为运动学模型和动力学模型, 然后基于模型进行控 制方面的研究, 其中控制方面可以简单分为开环控 制和闭环控制。

\subsection{1 运动学建模}

机器人运动学研究的是机器人的工作空间与 关节空间之间的映射关系, 目的是建立运动学模 型。运动学模型包括正运动学模型和逆运动学模 型两部分, 正运动学模型表示的是从关节空间到 工作空间的映射关系, 逆运动学模型则与之相反。 运动学模型是机器人运动控制的最基本的模型, 它的准确度会对机器人的运动分析和控制产生极 大影响。

对于球形机器人而言, 对其位置的描述方式较 为固定, 但是有多种不同的姿态描述方式, 例如战 强等利用欧拉角描述 BHQ-1 机器人的姿态, JOSHI 等运用四元数描述球形机器人的姿态。针对不同的 位姿描述方法, 可以基于旋量理论等对机器人建立 运动学模型, 得到球形机器人的速度雅克比矩阵, 然后通过广义逆解等方式得到机器人的逆运动学模 型。举例而言, 蔡尧等基于旋量理论和指数积方法 建立了 BHQ-1 的运动学模型, 并且基于 BP 神经网 络提出了一种求运动学逆解的方法。

对球形机器人建立起运动学模型之后, 往往需 要利用李代数理论运用 $\mathrm{CHOW}$ 定理或可控性秩条 件证明系统的可控性, 这在许多研究人员的论文中 均有体现, 例如 MURALIDHARAN 等、蔡尧等的 论文。

\subsection{2 动力学建模}

机器人动力学研究的是施加给机器人的力与机 器人产生的运动之间的关系, 包括正动力学和逆动 力学两部分内容。对于球形机器人而言, 基于运动 学模型的轨迹跟踪控制律以速度作为系统的输入 量, 但实际系统的输入往往是驱动力矩。另外, 在 机器人的高速和高精度控制方面, 机器人的动力学 特性是必须考虑的重要因素。球形机器人动力学建
模方法比较多, 其中应用最广泛的是拉格朗日方程, 包含 Euler-Lagrange 方程、Lagrange-Routh 方程等。 其他建模方法也有应用, 如 JOSHI 等用了达朗贝尔 方程, 战强、刘增波等用了 Boltzmann-Hamel 方程, 蔡尧等、孙汉旭等用了凯恩方程, MURALIDHARAN 等及 GAJBHIYE 等运用了 Euler-Poincaré 方程。

\subsection{3 开环控制}

开环控制指的是没有反馈信息的控制方式，或 者反馈信息对控制系统没有影响。在球形机器人开 发之初, 研究人员在建立模型之后往往通过开环控 制的方式来检验模型的正确性以及机器人的运动性 能。例如刘增波等针对 BHQ-2 的直线运动和圆周运 动两种情况, 基于机器人的运动学方程, 运用广义 逆的解法计算逆运动学解, 然后分别考虑直线和圆 周两种运动轨迹下的开环控制, 通过仿真和试验验 证了球形机器人运动速度逆解是正确的。但是在开 环控制下, 机器人的运动轨迹易受到外界噪声的干 扰, 运动误差会逐渐累积。因此要想获得高运动精 度, 必须对机器人进行闭环控制。

\subsection{4 闭环控制}

闭环控制是指根据被控对象的输出反馈来对控 制输入进行校正的控制方式, 利用系统输出状态的 实际测量值与预期值之间的差值作为反馈量，控制 系统基于该反馈发出新的控制指令来纠正偏差，消 除误差即可使被控对象按预期轨迹运动。球形机器 人的闭环控制可以分为多种情况, 例如对机器人进 行定点控制、坡面稳定控制、路径跟随控制以及轨 迹跟踪控制等。球形机器人作为一种移动机器人, 对其进行路径跟随或者轨迹跟踪控制是控制方面最 基础和最关键的内容, 其中路径跟随针对的仅是机 器人位置曲线的几何形状, 轨迹跟踪则是对机器人 状态量沿预期状态的实时跟踪控制。目前对于球形 机器人轨迹跟踪控制的研究多局限于对机器人的位 置实现轨迹跟踪, 对姿态进行轨迹跟踪控制的研究 相对较少。

大多数闭环控制方法研究是基于动力学模型 的。战强等基于 BHQ-2 的动力学模型, 利用反步法 设计控制律, 实现了对机器人位置以及方向角(机器 人沿坚直轴 $Z$ 轴的转角)的轨迹跟踪控制。郑明慧等 基于 BHQ-1 的运动学和动力学模型实现了路径跟 随控制, 并针对动力学模型的不准确性利用径向基 函数网络实现了对机器人位置的轨迹跟踪控制。

KAYACAN 等基于球形机器人的动力学模型, 利用 反馈线性化、模糊 PD 控制、模糊神经网络控制等 方法实现了闭环控制, 其反馈控制量为球形机器人 前向滚动的转角、速度以及加速度。少数研究人员 
基于运动学模型对闭环控制展开深入研究。蔡尧等 基于 BHQ-1 的运动学模型, 利用神经动力学 Shunting 模型实现了对机器人位置的轨迹跟踪控制。

近年来, 为了优化球形机器人的闭环控制, 也 有研究人员在球形机器人的环境感知能力等方面展 开研究。例如, 叶平等针对球形机器人因外部感知 能力弱而导致的控制精度不高的问题, 设计了一款 具有立体视觉的球形机器人 Visionbot, 立体视觉为 机器人的控制系统提供视觉反馈, 从而提高机器人 的运动控制性能。

\section{3 应用难点及展望}

基于对球形机器人的多年研究, 我们列出了目 前球形机器人研究的难点问题及可能的解决途径。

(1) 目前的动力学建模方法难以对球形机器人 建立准确的动力学模型, 基于不准确的模型提出的 控制方法难以保证控制准确性, 因此将其应用到实 际机器人时, 其控制效果难以达到预期。针对动力 学建模不准确的问题, 可以通过神经网络控制器对 动力学模型的不准确性进行补偿, 通过训练学习不 断对模型进行修正。

(2) 目前难以为球形机器人提供一个稳定的平 台以用于搭载设备。对于平面上运动的球体而言, 其姿态是十分不稳定的, 实现球形机器人运动过程 中的姿态控制一直是个难题。对于这一问题, 可以 从优化结构设计和优化控制方法两个方面进行改 进, 例如结构方面可以在球心处开辟空间设计一个 自稳定平台, 控制方面可以将三个姿态角状态量加 入到控制器中去。

(3) 目前对球形机器人的研究多局限于其在平 整地面或坡面上的应用, 越障能力较弱。这个问题 需要结合球形机器人的驱动方式来具体设计机器人 的越障方式, 例如摆锤驱动的机器人可通过摆锤的 高速转动实现机器人跳过障碍。

(4) 目前球形机器人对外部环境的感知能力较 弱, 难以在探测环境中独立工作。这个问题需要在 球形机器人具有稳定平台之后解决, 增加传感设备 以提高其感知能力, 从而丰富球形机器人的功能, 以其为载体完成具体任务。

\section{4 结论}

(1) 目前, 国内外对于球形机器人的研究重点 发生了转移。球形机器人产生之初, 国内外学者的 研究重点是球形机器人的机构设计, 但是目前研究 重点转移到了球形机器人的运动控制。

(2) 控制方法发生了变化。最初以研究机器人
的运动性能为主, 所以控制方法多是开环控制, 但 现在对球形机器人控制方面的研究多为闭环控制, 利用闭环控制实现路径跟随、轨迹跟踪等功能。另 外, 现在越来越多的研究将神经网络、机器学习等 理论应用到球形机器人的控制, 优化球形机器人的 控制。

(3) 对球形机器人的控制多基于动力学模型, 但目前的控制理论还不成熟。基于动力学模型进行 控制更为准确, 但是不同机器人的动力学模型是不 同的, 因此针对一个机器人设计的控制系统难以 应用于另一个机器人。另外就目前的研究现状而 言, 控制方面依然存在着许多问题, 尤其是姿态 控制方面。

(4) 球形机器人的环境感知能力得到提高。通 过将立体视觉等定位方法应用在球形机器人上, 机 器人的环境感知能力得到提高, 从机器人的状态反 馈方面提高了机器人的运动控制效果。

(5) 球形机器人的应用领域逐步扩大。近些年 来出现了水面球形机器人和水下球形机器人, 球形 机器人的应用环境已经从陆地扩展到了水中。

\section{参 考 文 献}

[1] HALME A, SCHONBERG T, WANG Y. Motion control of a spherical mobile robot[C]// 4th IEEE International Workshop on Advanced Motion Control, March 18-21, 1996, Mie University, Mie, Japan. IEEE, 1996: 259-264.

[2] BICCHI A, BALLUCHI A, PRATTICHIZZO D, et al. Introducing the "SPHERICLE": An experimental testbed for research and teaching in nonholonomy[C]// IEEE International Conference on Robotics and Automation, April 25-25, 1997, Albuquerque, New Mexico. IEEE, 1997: 2620-2625.

[3] Sphero | Connected Toys[EB/OL]. [2018-02-03]. http: //www.sphero.com/.

[4] KARAVAEV Y L, KILIN A A. The dynamics and control of a spherical robot with an internal omniwheel platform[J]. Regular \& Chaotic Dynamics, 2015, 20(2): 134-152.

[5] CHEMEL B, MUTSCHLER E, SCHEMPF H. Cyclops: Miniature robotic reconnaissance system[C]// IEEE International Conference on Robotics and Automation, May 10-15, 1999, Marriott Hotel, Renaissance Center, Detroit, Michigan. IEEE, 1999: 2298-2302.

[6] MUKHERJEE R, MINOR M A, PUKRUSHPAN J T. Simple motion planning strategies for spherobot: A spherical mobile robot[C]// IEEE Conference on Decision and Control, December 7-10, 1999, Phoenix, Arizona. IEEE, 1999: 2132-2137. 
[7] TOMIK F, NUDEHI S, FLYNN L L, et al. Design, fabrication and control of spherobot: A spherical mobile robot[J]. Journal of Intelligent \& Robotic Systems, 2012, 67(2): 117-131.

[8] AMIR H J A, MOJABI P. Introducing August: A novel strategy for an omnidirectional spherical rolling $\operatorname{robot}[\mathrm{C}] / /$ IEEE International Conference on Robotics and Automation, April 26 - May 1, 2004, Hilton New Orleans Riverside, New Orleans, Los Angeles. IEEE, 2004: 3527-3533.

[9] BHATTACHARYA S, AGRAWAL S K. Spherical rolling robot: A design and motion planning studies[J]. IEEE Transactions on Robotics \& Automation, 2000, 16(6): 835-839.

[10] JOSHI V A, BANAVAR R N. Motion analysis of a spherical mobile robot[J]. Robotica, 2009, 27(3): 343-353.

[11] JOSHI V A, BANAVAR R N, HIPPALGAONKAR R. Design and analysis of a spherical mobile robot[J]. Mechanism \& Machine Theory, 2010, 45(2): 130-136.

[12] MURALIDHARAN V , MAHINDRAKAR A D. Geometric controllability and stabilization of spherical robot dynamics[J]. IEEE Transactions on Automatic Control, 2015, 60(10): 2762-2767.

[13] SUGIYAMA Y, SHIOTSU A, YAMANAKA M, et al. Circular/spherical robots for crawling and jumping[C]// IEEE International Conference on Robotics and Automation, May 15-19, 2006, Hilton in Walt Disney World Resort, Orlando, Florida. IEEE, 2006: 3595-3600.

[14] WAIT K W, JACKSON P J, SMOOT L S. Selflocomotion of a spherical rolling robot using a novel deformable pneumatic method[C]// IEEE International Conference on Robotics and Automation, May 4-8, 2010, Anchorage, Alaska. IEEE, 2010: 3757-3762.

[15] OTANI T, URAKUBO T, MAEKAWA S, et al. Position and attitude control of a spherical rolling robot equipped with a gyro[C]// IEEE International Workshop on Advanced Motion Control, March 27-29, 2006, Istanbul, Turkey. IEEE, 2006: 416-421.

[16] URAKUBO $\mathrm{T}$, OSAWA $\mathrm{M}$, TAMAKI $\mathrm{H}$, et al. Development of a spherical rolling robot equipped with a gyro[C]// IEEE International Conference on Mechatronics and Automation, August 5-10, 2012, Chengdu, China. IEEE, 2012: 1602-1607.

[17] URAKUBO T, MONNO M, MAEKAWA $\mathrm{S}$, et al. Dynamic modeling and controller design for a spherical rolling robot equipped with a gyro[J]. IEEE Transactions on Control Systems Technology, 2016, 24(5): 1669-1679. //www.rotundus.se/.

[19] ERKAN K, BAYRAKTAROGLU Z Y, WOUTER S. Modeling and control of a spherical rolling robot: A decoupled dynamics approach[J]. Robotica, 2012, 30(4): 671-680

[20] KAYACAN E, KAYACAN E, RAMON H, et al. Adaptive neuro-fuzzy control of a spherical rolling robot using sliding-mode-control-theory-based online learning algorithm[J]. IEEE Transactions on Cybernetics, 2013, 43(1): 170.

[21] ROOZEGAR M, MAHJOOB M J, JAHROMI M. DP-based path planning of a spherical mobile robot in an environment with obstacles[J]. Journal of the Franklin Institute, 2014, 351(10): 4923-4938.

[22] ROOZEGAR M, MAHJOOB M J, JAHROMI M. Optimal motion planning and control of a nonholonomic spherical robot using dynamic programming approach: Simulation and experimental results[J]. Mechatronics, 2016, 39: 174-184.

[23] BELLMAN R. Dynamic programming and modern control theory $[\mathrm{J}]$. Proc. International Congress Math, 1965, 37(1): 65-82.

[24] ROOZEGAR M, MAHJOOB M J, ESFANDYARI M J, et al. XCS-based reinforcement learning algorithm for motion planning of a spherical mobile robot[J]. Applied Intelligence, 2016, 45(3): 1-11.

[25] ROOZEGAR M, MAHJOOB M J. Modelling and control of a non-holonomic pendulum-driven spherical robot moving on an inclined plane: Simulation and experimental results[J]. IET Control Theory \& Applications, 2017, 11(4): 541-549.

[26] GAJBHIYE S, BANAVAR R N. Geometric modeling and local controllability of a spherical mobile robot actuated by an internal pendulum $[\mathrm{J}]$. International Journal of Robust \& Nonlinear Control，2016，26(11): 2436-2454.

[27] SHEN J , SCHNEIDER D A , BLOCH A M. Controllability and motion planning of a multibody Chaplygin's sphere and Chaplygin's top[J]. International Journal of Robust \& Nonlinear Control, 2008， 18(9): 905-945.

[28] SHEN J, MCCLAMROCH N H, BLOCH A M. Local equilibrium controllability of multibody systems controlled via shape change[J]. IEEE Transactions on Automatic Control, 2004, 49(4): 506-520.

[29] LIN X, GUO S. Development of a spherical underwater robot equipped with multiple vectored water-jet-based thrusters[J]. Journal of Intelligent \& Robotic Systems, 2012, 67(3-4): 307-321. 
[30] LI Y, GUO S, YUE C. Preliminary concept of a novel spherical underwater robot[J]. International Journal of Mechatronics \& Automation, 2015, 5(1): 11-21.

[31] MAHBOUBI S, FAKHRABADI M M S, GHANBARI A. Design and implementation of a novel spherical mobile robot[J]. Journal of Intelligent \& Robotic Systems, 2013, 71(1): 43-64.

[32] HOGAN F R, FORBES J R. Modeling of spherical robots rolling on generic surfaces[J]. Multibody System Dynamics, 2015, 35(1): 91-109.

[33] DEJONG B P, KARADOGAN E, YELAMARTHI K, et al. Design and analysis of a four-pendulum omnidirectional spherical robot[J]. Journal of Intelligent \& Robotic Systems, 2017, 86(1): 1-13.

[34] 复杂机构与智能控制实验室 [EB/OL]. [2018-02-03]. http: //cmic.buaa.edu.cn/.

Complex Mechanism and Intelligent Control Laboratory [EB/OL]. [2018-02-03]. http: //cmic.buaa.edu.cn/.

[35] 战强. 一种新型的月球探测车运动机构 $[\mathrm{C}] / /$ 第二届月 球探测技术研讨会, 2001. 北京: 清华大学出版社, 2001: 328-330.

ZHAN Qiang. A novel moving mechanism for moon exploring vehicles[C]// The 2nd Workshop on Moon Exploring Technology, Beijing: Tsinghua University Press, 2001: 328-330.

[36] 战强, 贾川, 马晓辉, 等. 一种球形移动机器人的运动 性能分析[J]. 北京航空航天大学学报, 2005, 31(7): 744-747.

ZHAN Qiang, JIA Chuan, MA Xiaohui, et al. Analysis of moving capability of a spherical mobile robot[J]. Journal of Beijing University of Aeronautics and Astronautics, 2005, 31(7): 744-747.

[37] ZHAN Qiang, JIA Chuan, MA Xiaohui, et al. Mechanism design and motion analysis of a spherical mobile robot[J]. Chinese Journal of Mechanical Engineering, 2005, 18(4): $542-545$

[38] 战强, 蔡三龙, 刘增波. 一种球形探测机器人的电机控 制研究 [J]. 材料科学与工艺, 2006, 14(增刊): 96-100. ZHAN Qiang, CAI Sanlong, LIU Zengbo. Research on motor control of a spherical mobile robot for exploration[J]. Materials Science \& Technology, 2006, 14(Suppl.): 96-100.

[39] ZHAN Qiang, ZHOU Tingzhi, CHEN Ming, et al. Dynamic trajectory planning of a spherical mobile $\operatorname{robot}[\mathrm{C}] / /$ IEEE Conference on Robotics, Automation and Mechatronics, June 7-9, 2006, The Twin Towers Hotel, Bangkok, Thailand. IEEE, 2006: 1-6.

[40] CHEN Ming, ZHAN Qiang, LIU Zengbo, et al. Optimal trajectory planning based on Hamiltonian function of a spherical mobile robot[J]. High Technology Letters, 2008, 14(3): 289-293.

[41] 刘增波, 战强, 蔡尧. 一种环境探测球形移动机器人的 运动控制[J]. 航空学报, 2008, 29(6): 1673-1679.

LIU Zengbo, ZHAN Qiang, CAI Yao. Motion control of a spherical mobile robot for environment exploration[J]. Acta Aeronautica Et Astronautica Sinica, 2008, 29(6): 1673-1679.

[42] ZHAN Qiang, CAI Yao, LIU Zengbo. Near-optimal trajectory planning of a spherical mobile robot for environment exploration[C]// IEEE Conference on Robotics, Automation and Mechatronics, September 21-24, 2008, Chengdu, China. IEEE, 2008: 84-89.

[43] ZHAN Qiang, LIU Zengbo, CAI Yao. A back-stepping based trajectory tracking controller for a non-chained nonholonomic spherical robot $[\mathrm{J}]$. Chinese Journal of Aeronautics, 2008, 21(5): 472-480.

[44] CAI Yao, ZHAN Qiang, XI Xi, et al. Inverse kinematics identification of a spherical robot based on BP neural networks[C]// IEEE Conference on Industrial Electronics and Applications, June 21-23, 2011, Beijing, China. IEEE, 2011: 2114-2119.

[45] CAI Yao, ZHAN Qiang, YAN Caixia. Two-state trajectory tracking control of a spherical robot using neurodynamics[J]. Robotica, 2012, 30(2): 195-203.

[46] CAI Yao, ZHAN Qiang, XI Xi. Neural network control for the linear motion of a spherical mobile robot[J]. International Journal of Advanced Robotic Systems, 2011, 8(4): 79-87.

[47] ZHAN Qiang, CHI Xing, XI Xi. Linear motion control of an underactuated spherical mobile robot[J]. Applied Mechanics \& Materials, 2014, 644-650, 351-355.

[48] CAI Yao, ZHAN Qiang, XI Xi. Path tracking control of a spherical mobile robot[J]. Mechanism \& Machine Theory, 2012, 51(5): 58-73.

[49] ZHENG Minghui, ZHAN Qiang, LIU Jinkun, et al. Control of a spherical robot: Path following based on nonholonomic kinematics and dynamics[J]. Chinese Journal of Aeronautics, 2011, 24(3): 337-345.

[50] ZHENG Minghui, ZHAN Qiang, LIU Jinkun, et al. Trajectory tracking of a spherical robot based on an RBF neural network[J]. Advanced Materials Research, 2012, 383-390, 631-637.

[51] ZHAN Qiang, CAI Yao, YAN Caixia. Design, analysis and experiments of an omni-directional spherical robot[C]// IEEE International Conference on Robotics and Automation, May 9-13, 2011, Shanghai, China. IEEE, 
2011: 4921-4926.

[52] ZHAN Qiang, LIU Zening. A deformable spherical mobile robot[J]. Applied Mechanics \& Materials, 2014, 590(590): 422-426.

[53] 战强, 刘泽宁, 张超. 一种可变形球形探测机器人: 中 国, CN104002886A[P]. 2014-08-27.

ZHAN Qiang, LIU Zening, ZHANG Chao. A deformable spherical mobile robot for exploration : China , CN104002886A[P]. 2014-08-27.

[54] 肖爱平, 孙汉旭, 廖启征, 等. 一种球形机器人的设计 与原理分析 $[\mathrm{J}]$. 机电产品开发与创新, 2004, 17(1): 14-16.

XIAO Aiping, SUN Hanxu, LIAO Qizheng, et al. Design and principle analysis of a spherical robot[J]. DeveIopment \& Innovation of Machinery \& Electrical Products, 2004, 17(1): 14-16.

[55] 孙汉旭, 肖爱平, 贾庆轩, 等. 二驱动球形机器人的全 方位运动特性分析 [J]. 北京航空航天大学学报, 2005, 31(7): 735-739.

SUN Hanxu, XIAO Aiping, JIA Qingxuan, et al. Omnidirectional kinematics analysis on bi-driver spherical robot[J]. Journal of Beijing University of Aeronautics and Astronautics, 2005, 31(7): 735-739.

[56] 王亮清, 孙汉旭, 贾庆轩. 球形机器人的爬坡与弹跳能 力 $[\mathrm{J}]$. 北京邮电大学学报, $2007,30(2)$ : 11-14.

WANG Qingliang, SUN Hanxu, JIA Qingxuan. Research on the climbing and jumping of a spherical rolling robot[J]. Journal of Beijing University of Posts and Telecommunications, 2007, 30(2): 11-14.

[57] 王亮清, 孙汉旭, 贾庆轩. 球形机器人的圆周运动分析 [J]. 机器人, 2007, 29(1): 56-60.

WANG Qingliang, SUN Hanxu, JIA Qingxuan. Circle motion analysis on a spherical robot[J]. Robot, 2007, 29(1): $56-60$.

[58] LIU Daliang, SUN Hanxu, JIA Qingxuan, et al. Motion control of a spherical mobile robot by feedback linearization $[\mathrm{C}] / /$ World Congress on Intelligent Control and Automation, June 25-27, 2008, Chongqing, China. 2008: 965-970.

[59] 孙汉旭, 王亮清, 贾庆轩, 等. BYQ-3 球形机器人的动 力学模型 $[J]$. 机械工程学报, 2009, 45(10): 8-14.

SUN Hanxu, WANG Qingliang, JIA Qingxuan, et al. Dynamic model of the BYQ-3 spherical robot[J]. Journal of Mechanical Engineering, 2009， 45(10): 8-14.

[60] 李团结, 张学锋, 陈永琴. 一种全向滚动球形机器人的 运动分析与轨迹规划 $[\mathrm{J}]$. 西安电子科技大学学报(自然 科学版), 2007, 34(1): 29-33.

LI Tuanjie, ZHANG Xuefeng, CHEN Yongqin. Motion analysis and trajectory planning of a spherical omnidirectional rolling $\operatorname{robot}[\mathrm{J}]$. Journal of Xidian University, 2007, 34(1): 29-33.

[61] 李团结, 严天宏, 张学锋. 一种全向滚动球形机器人的 动力学分析与仿真 $[\mathrm{J}]$. 西安电子科技大学学报, 2007 , 34(3): 4239-4242.

LI Tuanjie, YAN Tianhong, ZHANG Xuefeng. Dynamic analysis and simulation of a spherical omnidirectional rolling robot[J]. Journal of Xidian University, 2007, 34(3): 4239-4242.

[62] 岳明, 邓宗全. 基于状态观测器的球形机器人状态反馈 控制系统设计 [J]. 光学精密工程, 2007, 15(6): 878-883. YUE Ming, DENG Zongquan. Design of state feedback control system for spherical robot based on state observer[J]. Optics and Precision Engineering, 2007, 15(6): 878-883.

[63] YUE Ming, LIU Baoyin, AN Cong, et al. Extended state observer-based adaptive hierarchical sliding mode control for longitudinal movement of a spherical robot[J]. Nonlinear Dynamics， 2014， 78(2): 1233-1244.

[64] 赵勃, 王鹏飞, 孙立宁, 等. 双偏心质量块驱动球形机器 人的直线运动控制[J]. 机械工程学报, 2011, 47(11): 1-6. ZHAO Bo, WANG Pengfei, SUN Lining, et al. Linear motion control of two-pendulums-driven spherical $\operatorname{robot}[\mathrm{J}]$. Journal of Mechanical Engineering, 2011, 47(11): $1-6$.

[65] 杨伟, 李健, 肖起阳, 等. 球形机器人的仿真与实验测 试分析[J]. 制造业自动化，2017，39(8)：26-29.

YANG Wei, LI Jian, XIAO Qiyang, et al. The simulation and experimental analysis of a spherical robot[J]. Manufacturing Automation, 2017, 39(8): 26-29.

[66] 兰晓娟, 孙汉旭, 贾庆轩. 水下球形机器人 BYSQ-2 的 原理与动力学分析 $[\mathrm{J}]$. 北京邮电大学学报, 2010,33(3): 20-23.

LAN Xiaojuan, SUN Hanxu, JIA Qingxuan. Principle and dynamic analysis of a new-type spherical underwater vehicle[J]. Journal of Beijing University of Posts and Telecommunications, 2010, 33(3): 20-23.

[67] 李艳生, 孙汉旭, 贾庆轩, 等. 一种摆式球形水下机器 人水底滚动特性分析 $[\mathrm{J}]$. 中南大学学报, 2016, 47(11): 3664-3669.

LI Yansheng, SUN Hanxu, JIA Qingxuan, et al. Analysis of rolling characteristics at water bottom for spherical robot with heavy pendulums[J]. Journal of Central South University, 2016, 47(11): 3664-3669.

[68] CHEN W H, CHEN C P, TSAI J S, et al. Design and implementation of a ball-driven omnidirectional spherical robot[J]. Mechanism \& Machine Theory, 2013， 68(68): 
35-48.

[69] 叶平, 韩亮亮, 张天石, 等. 具有立体视觉的球形机器 人及其运动控制 [J]. 机械工程学报, 2013, 49(11): 8-15. YE Ping, HAN Liangliang, ZHANG Tianshi, et al. Design and motion control of ball-shaped robot with stereo vision[J]. Journal of Mechanical Engineering, 2013, 49(11): 8-15.

[70] YE Ping, SUN Hanxu, QIU Zhongjiang, et al. Design and motion control of a spherical robot with stereovision[C]// Industrial Electronics and Applications, 5-7 June, 2016, Hefei, China. IEEE, 2016: 1276-1282.

作者简介: 战强(通信作者), 男, 1972 年出生, 博士, 教授, 博士研究 生导师。主要研究方向为机器人机构设计与控制、球形机器人和仿生灵 巧手。

E-mail: qzhan@buaa.edu.cn

李伟, 男, 1994 年出生, 硕士研究生。主要研究方向为机器人控制。 E-mail: li.wei.antoine@foxmail.com

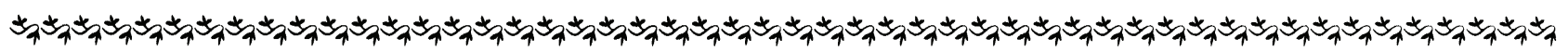

第 8 届上银优秀机械博士论文奖一一佳作奖

\section{整体叶盘振动特性分析与硬涂层阻尼减振设计研究}

作 者: 陈玉刚

毕业学校: 大连理工大学

指导教师: 韩清凯

整体叶盘是为了提高航空发动机性能而采用的一种新型结构部件, 在当前先进航空发动机结构中得到了高度重视和成功 应用。整体叶盘的振动及疲劳破坏问题极为突出: 容易出现盘片刚度相近而导致发生耦合振动; 无榫结构形式降低了振动阻 尼能力, 使得其在恶劣工作环境条件下容易处于大振动和高动应力状态; 整体叶盘发生疲劳破坏后的修复难度很高。

针对整体叶盘减振和抗振动疲劳的需求, 本文采用解析分析、数值计算与试验测试相结合的方法, 研究了考虑结构特征 的整体叶盘动力学建模方法, 明确了其耦合共振机理; 提出了整体叶盘振动抑制与抗振动疲劳的硬涂层阻尼技术, 在掌握其 结构振动抑制与抗疲劳破坏机理及影响规律的基础上进行了减振优化设计; 针对失谐整体叶盘的振动局部化、振动响应放大 和振动疲劳破坏问题提出了硬涂层主动失谐设计方法。

本文所提出的整体叶盘振动抑制和抗疲劳的硬涂层阻尼技术具有创新性; 硬涂层阻尼对整体叶盘结构振动抑制与抗瘦劳 破坏机理及影响规律、多目标动态优化设计方法与结果、硬涂层主动失谐设计的方法和结果具有合理性, 并通过了模型试验 验证。研究成果可以为提高先进航空发动机整体叶盘的动力学设计水平、提高其运行安全性提供理论支撑和技术指导。 